\title{
FORMAS DE LEITO E TRANSPORTE DE CARGA DE FUNDO DO ALTO RIO PARANÁ ${ }^{1}$
}

\author{
Débora Pinto Martins \\ Universidade Estadual de Maringá, GEMA - Grupo de Estudos Multidisciplinares do Ambiente, Av. Colombo, 5790. 87020-900 \\ Maringá, $P R$ \\ deby_martins@yahoo.com.br \\ José Cândido Stevaux \\ Universidade de Guarulhos, Lab. Geociências, Praça Tereza Cristina, 1, 07023-070, Guarulhos, SP \\ jcstevaux@uem.br
}

\begin{abstract}
Resumo
O presente trabalho estima o transporte de carga de fundo no alto rio Paraná em Porto São José, PR (22 $45^{\prime} 52^{\prime}$ 'S e $53^{\circ} 10^{\prime} 34^{\prime \prime} \mathrm{W}$ ), num trecho de aproximadamente $2 \mathrm{~km}$ a jusante da Usina Hidrelétrica Engenheiro Sérgio Motta (Porto Primavera), onde o rio Paraná apresenta canal único com largura média de $1.200 \mathrm{~m}$ e vazão média anual é de $8.912 \mathrm{~m}^{3} / \mathrm{s}$. O cálculo da carga de fundo do canal foi baseado na determinação do tamanho das formas de leito e de sua velocidade de deslocamento linear, obtidos a partir de sucessivos levantamentos batimétricos. Foram realizadas três campanhas de levantamentos ecobatimétricos em diferentes momentos do ciclo hidrológico do rio, sendo cada campanha constituída por dois levantamentos separados em intervalos de 16 a 20 dias. O cálculo da carga de fundo baseou-se equação: $\mathrm{Cf}=(1-\mathrm{p}) \mathrm{H} \mathrm{k} \mathrm{Ud}$ onde $\mathrm{p}$ é a porosidade (adimensional), $\mathrm{H}$ a altura média das formas de leito $(\mathrm{m}), \mathrm{k}$ o coeficiente morfométrico das formas de leito (adimensional) e Ud a velocidade de deslocamento das formas de leito (m/dia). As dunas são as formas de leito predominantes neste trecho do rio Paraná, com comprimento entre 50 e $100 \mathrm{~m}$ (média de $70,68 \mathrm{~m}$ ) e altura de 0,80 a 2,20 m (média de 1,30 m). A velocidade média de deslocamento linear das formas de fundo foi de $56,8 \mathrm{~m} / \mathrm{mês}$, para o período de maior vazão; e $45,0 \mathrm{~m} / \mathrm{mês}$ para o período de menor vazão. O transporte médio de carga e fundo no rio Paraná foi estimado em 2.820,6 ton/dia, o que corresponde a 1.029.300 ton/ano. Comparando com dados anteriores, os autores sugerem que o transporte hidrossedimentar de fundo no trecho de canal estudado está sofrendo interferência da barragem de Porto Primavera.
\end{abstract}

Palavras-chave: Rio Paraná; Formas de leito; Carga de fundo; Impacto de barragem.

\begin{abstract}
A new method for bedload discharge estimative is presented and applied in a $2 \mathrm{~km}$ reach of the upper Paraná River (Porto

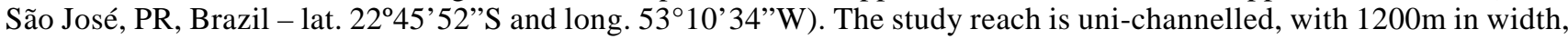
annual average discharge of $8,912 \mathrm{~m}^{3} / \mathrm{s}$, and is situated about $28 \mathrm{~km}$ downstream of the Porto Primavera Hydroelectric Plant. This method bases on the determination of bedform size, shape and velocity of linear displacement, acquired from echo-bathymetric longitudinal profiles performed in intervals of 16 to 20 days. For the present analysis, three echobathymetric surveys were taken in three moments of the Paraná River's hydrological cycle. The calculation of the bottom load was based equation: $\mathrm{Cf}=(1-\mathrm{p}) \mathrm{H} \mathrm{k} \mathrm{Ud}$ where: $\mathrm{p}$ - is porosity (dimensionless), $\mathrm{H}$ - medium height in the bed ways $(\mathrm{m})$, $\mathrm{k}$ - duna morphometric coefficient (dimensionless), and $\mathrm{Ud}$ - dune displacement speed ( $\mathrm{m} /$ day). The predominant bed forms found were dunes with medium length of $65 \mathrm{~m}$ and height varying between $0.80 \mathrm{~m}$ and $2.20 \mathrm{~m}$. The mean speed of linear displacement was $1.8 \mathrm{~m} /$ day $(56.8 \mathrm{~m} / \mathrm{month})$ for the period of larger debit and $1.5 \mathrm{~m} /$ day $(45.0 \mathrm{~m} / \mathrm{month})$ for the smaller one. The bed load discharge estimated to the Paraná River was 2,820.6 ton/day (1,029,300 ton/year).
\end{abstract}

Key Words: Paraná River; Bedload discharge; Bedform.

\section{Introdução}

Problemas comuns em grandes sistemas fluviais como a operacionalidade de hidrovias, instalação de portos, construção de praias artificiais e extração de areia para construção civil, estão diretamente relacionados ao conhecimento da característica, dinâmica e distribuição da carga de fundo do canal. Se por um lado os métodos para medição de carga suspensa são abundantes na literatura, a quantificação da carga de fundo constitui ainda uma dificuldade a ser enfrentada. 
Nos cálculos da carga de fundo é comum o uso de fórmulas que variam desde métodos puramente empíricos, até modelagens teóricas complexas. Em geral, tais metodologias tendem a associar a magnitude do transporte a parâmetros como tensão de cisalhamento, potência do escoamento e turbulência, considerando estes como os principais agentes condicionantes do transporte de sedimentos no fundo do canal.

Entre 1986 e 1989, a Itaipu Binacional desenvolveu um projeto sistemático de avaliação da carga transportada pelo rio Paraná e seus principais afluentes, abrangendo o trecho compreendido entre a foz do rio Paranapanema e o remanso do reservatório de Itaipu. A descarga sólida verificada no trecho estudado, para o período de 1986 a 1988 , foi de $30 \mathrm{x}$ $10^{6}$ ton/ano, sendo $27 \times 10^{6}$ ton/ano correspondente à carga suspensa e $3 \times 10^{6}$ ton/ano correspondente à carga de fundo (ITAIPU BINACIONAL, 1990). Neste caso a carga de fundo foi obtida de forma indireta, pela relação: $\mathrm{Cf}=0,2 \mathrm{Cs}$ onde, $\mathrm{Cf}$ é a carga de fundo e Cs a carga suspensa.

Estudos relativos à hidrossedimentologia da carga de fundo no rio Paraná são pouco comuns, sendo mais desenvolvidos em seu trecho médio, entre Corrientes e Santa Fé, Argentina (LIMA et al., 1990; AMASLER \& GAUDIN, 1994; AMASLER \& SCHREIDER, 1999) e baseiam-se no trabalho pioneiro de Stuckrath (1969). No alto curso do rio Paraná os estudos abordaram a composição e textura (SANTOS et al., 1992, SANTOS, 1991, SOUZA FILHO, 1993), a morfologia (STEVAUX, 1994) e a migração das formas de leito ao longo do talvegue (STEVAUX et al., 1995). Mais recentemente, Crispim (2001) avaliou, nesse mesmo trecho, as alterações introduzidas na dinâmica da carga de fundo, pela construção da barragem de Porto Primavera, como também foram desenvolvidos estudos da dinâmica da carga de fundo em relação à comunidade bêntica do canal (STEVAUX \& TAKEDA, 1995).

A proposta do presente trabalho é a aplicação de uma metodologia ainda pouco explorada para quantificação da carga de fundo em canais fluviais de grande magnitude. Nesta metodologia, o transporte de sedimentos é determinado com base no tamanho das formas de leito e na sua velocidade de deslocamento linear ao longo de perfis longitudinais obtidos por meio de levantamentos batimétricos sucessivos (STUCKRATH, 1969).

O presente estudo tem importância não apenas metodológica, como também, irá subsidiar os estudos de impacto e controle ambiental no único trecho brasileiro do rio Paraná, que ainda se encontra em estado natural, sem barragens. Além disso, o segmento constitui uma área de proteção ambiental (Parque Nacional do Ivinhema) que sofre o estresse das seguintes atividades: a) navegação comercial (Hidrovia do rio Paraná), b) controle de descarga promovido pela represa de Porto Primavera a montante, c) crescente instalação de empreendimentos de recreação e lazer (pes- ca, balneário, condomínios de veraneio e esportes aquáticos) e d) pela intensa exploração de areia para construção civil.

\section{1. Área de estudo}

A bacia do rio da Prata é constituída de três grandes sistemas fluviais: Paraná, Paraguai e Uruguai, entendendose por toda região centro-sul da América do Sul, drenando uma área de $3.100 .000 \mathrm{~km}^{2}$, estando 45,6\% em território brasileiro, $29,7 \%$ na Argentina, $13,2 \%$ no Paraguai, 6,6\% na Bolívia e $4,8 \%$ no Uruguai. O rio Paraná, principal canal fluvial da bacia do rio da Prata, percorre uma distância aproximada de $2.738 \mathrm{~km}$ desde a sua nascente, na confluência dos rios Grande e Paranaíba, até sua foz, no estuário do rio da Prata (ORFEO \& STEVAUX, 2002). Dos $811 \mathrm{~km}$ do rio em território brasileiro, apenas num trecho de cerca de $200 \mathrm{~km}$, entre a represa de Porto Primavera e o remanso do lago de Itaipu, o rio se apresenta nas condições naturais (não represado).

Nesse trecho o segmento estudado tem dois quilômetros de comprimento e está localizado nas proximidades de

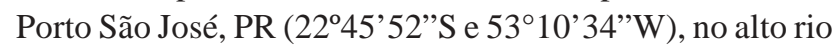
Paraná. Consiste de um segmento com canal único, que sofre influência direta das barragens de Porto Primavera, localizada $35 \mathrm{~km}$ a montante e da barragem Rosana, no rio Paranapanema, $46 \mathrm{~km}$ a montante (Figura 1).

$\mathrm{Na}$ margem esquerda do segmento de estudo se encontra a Estação Fluviométrica Porto São José, em operação desde 1964, e onde foram obtidos os dados hidrométricos utilizados no presente trabalho. Nesta estação, a vazão média anual é de $8.912 \mathrm{~m}^{3} / \mathrm{s}$ (para a série completa), com valores extremos de $33.740 \mathrm{~m}^{3} / \mathrm{s}$ (em 18/02/1983) e $2.551 \mathrm{~m}^{3} / \mathrm{s}$ (em 22/09/1969).

\section{Metodologia}

O método do deslocamento de dunas, desenvolvido primeiramente por Stuckrath (1969) e aprimorado por Amsler \& Gaudin (1994), permite medir indiretamente a carga de fundo em rios aluviais com o fundo coberto por dunas. Neste método o cálculo da carga de fundo de um canal, em última análise, é obtido pela determinação do tamanho das dunas e de sua velocidade de deslocamento ao longo do canal do rio, obtidas pela comparação de dois levantamentos consecutivos de perfis longitudinais (Figura 2).

Hubbel (1964), Simons et al. (1965) e Fredsøe (1981) demonstraram que, num rio aluvial de fluxo permanente e aproximadamente uniforme e que percorre um fundo móvel com formas de leito desenvolvidas, a carga sedimentar de fundo pode ser determinada pela equação: $\mathrm{Cf}=(1-\mathrm{p}) \mathrm{H} \mathrm{k}$ ud Eq. 1 onde: $\mathrm{p}$ é a porosidade o material de fundo, $\mathrm{H}$ é a altura média das dunas, $\mathrm{k}$ é o coeficiente de forma das dunas e ud a velocidade de deslocamento das dunas. 


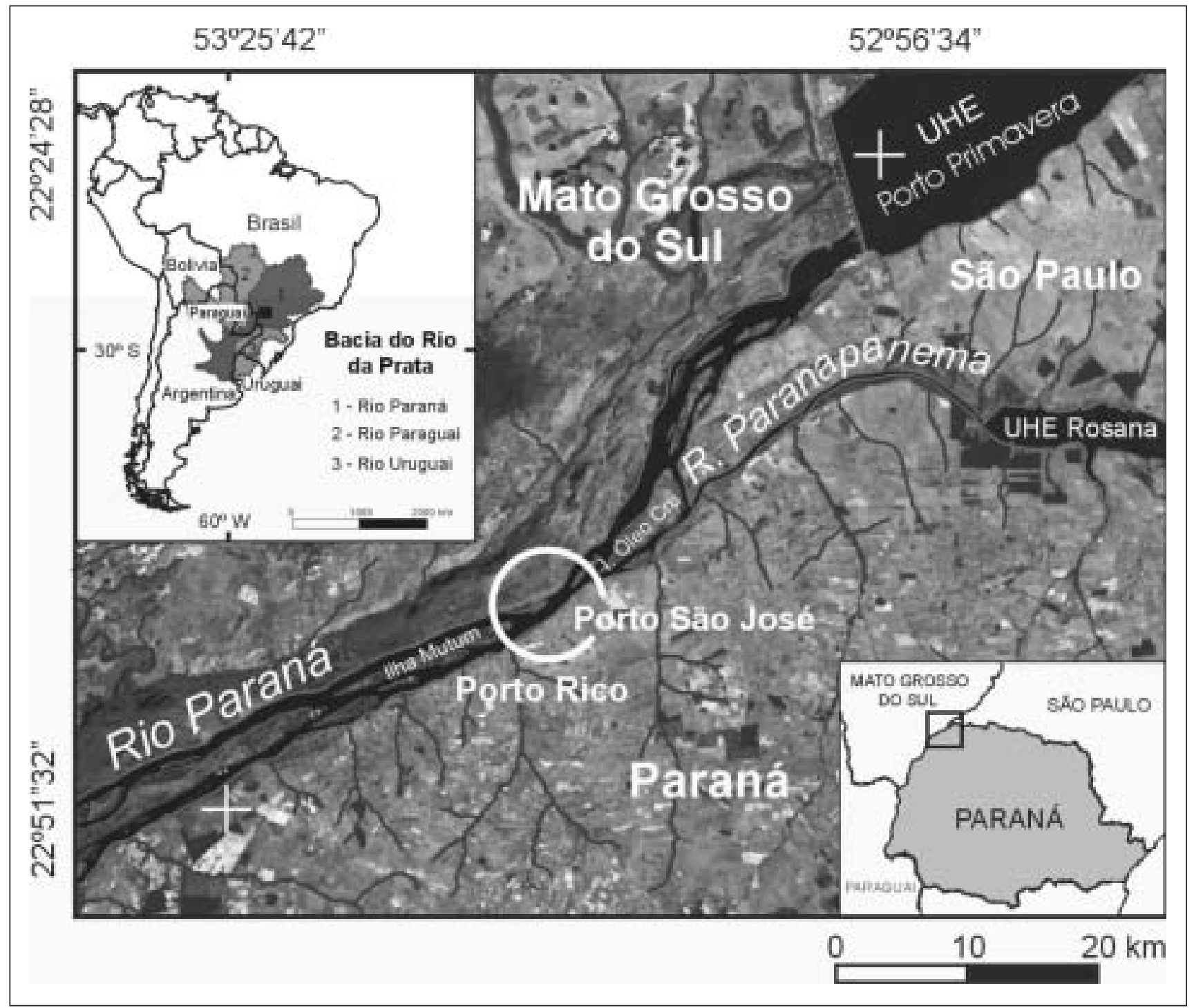

Figura 1: Localização da área de estudo (Imagem LANDSAT, 1999).

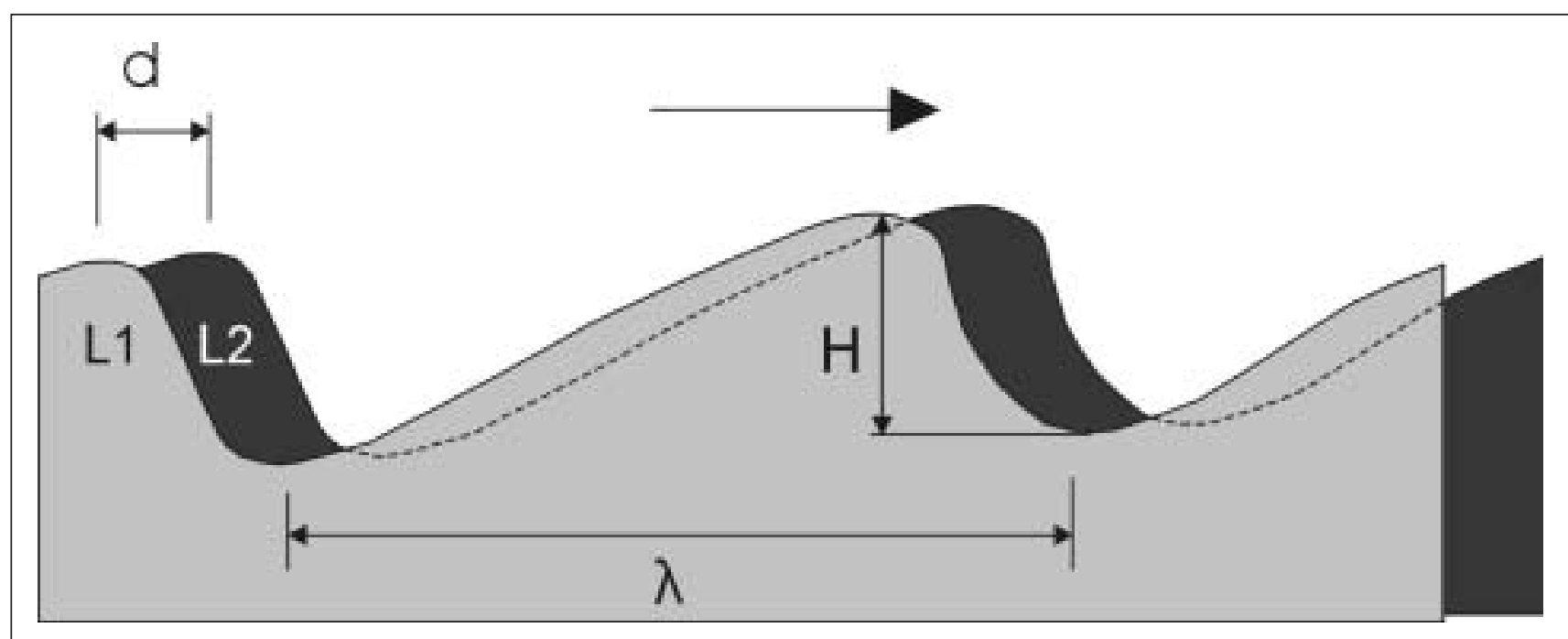

Figura 2: Duna típica de uma corrente aluvial em dois momentos diferentes (L1, cinza e L2, preto). A velocidade de migração é dada pela razão entre o deslocamento da duna (d) pelo intervalo de tempo entre dois levantamento. H e 1 são respectivamente a altura e o comprimento da duna. A seta indica a direção do fluxo. (Modificado de Amsler et al., 2000). 


\subsection{Obtenção dos perfis batimétricos}

Esta é a fase mais importante do trabalho e deve considerar duas questões: 1) qual o melhor período para realização das campanhas e 2) qual o intervalo temporal entre os dois levantamentos. No primeiro caso deve-se considerar o hidrograma do rio numa série histórica mais representativa possível. Assim, é interessante avaliar o transporte em períodos de águas baixas e nos períodos de cheias. O intervalo entre os dois levantamentos dependerá da velocidade de deslocamento das dunas, principalmente, do seu potencial para preservação das formas. Dessa forma, em rios ainda desconhecidos, é interessante fazer-se um ensaio com os intervalos entre os levantamentos gradativamente aumentados. No rio Araguaia $\left(\mathrm{Q}=6.429 \mathrm{~m}^{3} / \mathrm{s}\right)$, Silva (2002) usou-se um intervalo de seis dias. Por sua vez, Amasler \& Gaudin (1994) utilizaram um intervalo de 10 a 15 dias no baixo curso do rio Paraná $\left(\mathrm{Q}=16.900 \mathrm{~m}^{3} / \mathrm{s}\right)$.

Também é necessário estabelecer o número de perfis longitudinais a serem elaborados. Geralmente, pode-se considerar que ao menos um perfil deverá ser feito no talvegue e mais um próximo a cada margem do canal. É importante salientar que um número maior de perfis dependerá da largura e da morfologia da seção transversal do canal estudado. Em muitos casos é aconselhada a elaboração de perfis de apoio levantados paralelamente aos perfis principais. Os perfis de apoio têm a finalidade de se estimar a preservação ou a variação lateral das dunas.

\subsection{Tratamento dos dados}

Primeiramente serão processados os dados referentes aos perfis de apoio (identificados por letras minúsculas $\mathrm{a}, \mathrm{b}$ etc.) que deverão ser sintetizados nos perfis principais (identificados por números P1, P2 etc.). Numa segunda fase, os dados dos perfis principais são agrupados para produzirem os valores referentes ao canal. As etapas de tratamento de dados, descritas a seguir, obedecem a essa sequiência.

1. quantificação da altura $\left(\mathbf{H}_{\mathrm{Pla}}\right)$ e da distância caminhada pelas dunas $\left(\mathbf{d}_{\text {pla }}\right)$ nos perfis de apoio $\mathrm{P}_{1 \mathrm{a}}, \mathrm{P}_{1 \mathrm{~b}}, \mathrm{P}_{1 \mathrm{c}}$ conforme as equações:

$$
\begin{array}{ll}
\mathrm{H}_{\mathrm{Pla}}=\left(\Sigma \mathrm{H}_{\mathrm{i}}\right) / \mathrm{n} & \text { Eq. } 2 \\
\mathrm{~d}_{\mathrm{pla}}=\left(\Sigma \mathrm{d}_{\mathrm{i}}\right) / \mathrm{n} & \text { Eq. } 3
\end{array}
$$

onde, $\mathbf{H}_{\mathrm{pla}}$ é a altura média das dunas no perfil de apoio $\mathrm{P}_{1 \mathrm{a}}, \Sigma \mathbf{H}_{\mathrm{i}}$ é a somatória das alturas das dunas de $\mathrm{P}_{1 \mathrm{a}}, \mathrm{n}$ número de dunas de $\mathrm{P}_{1 \mathrm{a}}$, $\mathbf{d}_{\mathrm{pla}}$ é a distancia média caminhada pelas dunas de $\mathrm{P}_{1 \mathrm{a}}$ durante os dois levantamentos, $\Sigma \mathbf{d}_{\mathrm{i}}$ é a somatória da distância caminhada por cada duna do $\mathrm{P}_{1 \mathrm{a}}$ entre os dois levantamentos (Figura 2). Esses cálculos foram também efetuados para todos perfis de apoio;

2. quantificação dos valores de $\mathbf{H}_{\mathrm{p} 1}$ e $\mathbf{d}_{\mathrm{p} 1}$ para cada um dos perfis principais $\left(\mathrm{P}_{1}, \mathrm{P}_{2} \mathrm{P}_{3}\right.$ e $\left.\mathrm{P}_{4}\right)$ dada pela média dos valores obtidos em cada perfil de apoio (no caso três perfis de apoio):

$$
\begin{array}{ll}
\mathrm{H}_{\mathrm{pl}}=\left(\mathrm{H}_{\mathrm{pla}}+\mathrm{H}_{\mathrm{plb}}+\mathrm{H}_{\mathrm{plc}}\right) / 3 & \text { Eq. } 4 \\
\mathrm{~d}_{\mathrm{p} 1}=\left(\mathrm{d}_{\mathrm{pla}}+\mathrm{d}_{\mathrm{plb}}+\mathrm{d}_{\mathrm{plc}}\right) / 3 & \text { Eq. } 5
\end{array}
$$

3. cálculo da velocidade de deslocamento médio para cada perfil principal $\left(\mathbf{u d}_{\mathrm{p} 1}\right)$ :

$$
\mathrm{zud}_{\mathrm{p} 1}=\mathrm{d}_{\mathrm{p} 1} \Delta \mathrm{t}
$$

onde, "t é o intervalo em dias entre os dois levantamentos batimétricos; o mesmo procedimento para os outros perfis $\left(\mathbf{u d}_{\mathrm{p} 2,} \mathbf{u} \mathbf{d}_{\mathrm{p} 3} \mathrm{e} \mathbf{u \mathbf { d } _ { \mathrm { p } 4 }}\right)$;

4. cálculo da carga de fundo linear $\left(\mathbf{C f l}_{\mathrm{P} 1}\right)$ para cada perfil principal, sendo o resultado dado $\mathrm{em}^{2} /$ dia:

$$
\mathrm{Cfl}_{\mathrm{P} 1}=(1-\mathrm{p}) \mathrm{H}_{\mathrm{p} 1} \mathrm{kud}_{\mathrm{p} 1} \quad \text { Eq. } 7
$$

(p e k já foram apresentados anteriormente)

5. a seguir a carga de fundo linear é ponderada pela abrangência (em metros) de cada perfil no canal (Figura 3) obtendo-se a carga de fundo de cada perfil principal dada em $\mathrm{m}^{3} / \mathrm{dia}$ :

$$
\mathrm{Cf}_{\mathrm{p} 1}=\mathrm{Cfl}_{\mathrm{pl}} \mathrm{L}_{\mathrm{p} 1} \quad \text { Eq. } 8
$$
perfil;

onde, $\mathbf{L}_{\mathrm{p} 1}$ é a largura da faixa de abrangência de cada

6. a Cf de cada perfil é somada para se obter a carga total de sedimento de para a seção:

$$
\mathrm{Cf}_{\text {total }}=\mathrm{Cf}_{\mathrm{p} 1}+\mathrm{Cf}_{\mathrm{p} 2}+\mathrm{Cf}_{\mathrm{p} 3}+\mathrm{Cf}_{\mathrm{p} 4} \quad \text { Eq. } 9
$$

Multiplicando-se este valor pelo peso específico da areia $\left(2.650 \mathrm{~kg} / \mathrm{m}^{3}\right)$, obtêm-se o peso do material transportado para o período analisado.

\section{Cálculo da carga de fundo do Rio Paraná}

Neste estudo foram realizadas três campanhas de campo, cada uma composta por dois levantamentos ecobatimétricos, separados por intervalos de 16 a 20 dias. A escolha do intervalo ideal para a melhor caracterização do deslocamento das dunas, sem que estas se descaracterizassem, foi estabelecido baseando-se nos levantamentos realizados por Stevaux \& Takeda (1995).

As campanhas forma realizadas em nov-dez/2002, $\left(\mathrm{Q}_{\mathrm{m}}=\right.$ $\left.6.256,60 \mathrm{~m}^{3} / \mathrm{s}\right)$; jun-jul $/ 2003\left(\mathrm{Q}_{\mathrm{m}}=6.761,75 \mathrm{~m}^{3} / \mathrm{s}\right)$ e nov-dez/ $2003\left(\mathrm{Q}_{\mathrm{m}}=7.625,00 \mathrm{~m}^{3} / \mathrm{s}\right)$. A análise do hidrograma do período estudado (Figura 4) mostra que as campanhas foram de- 


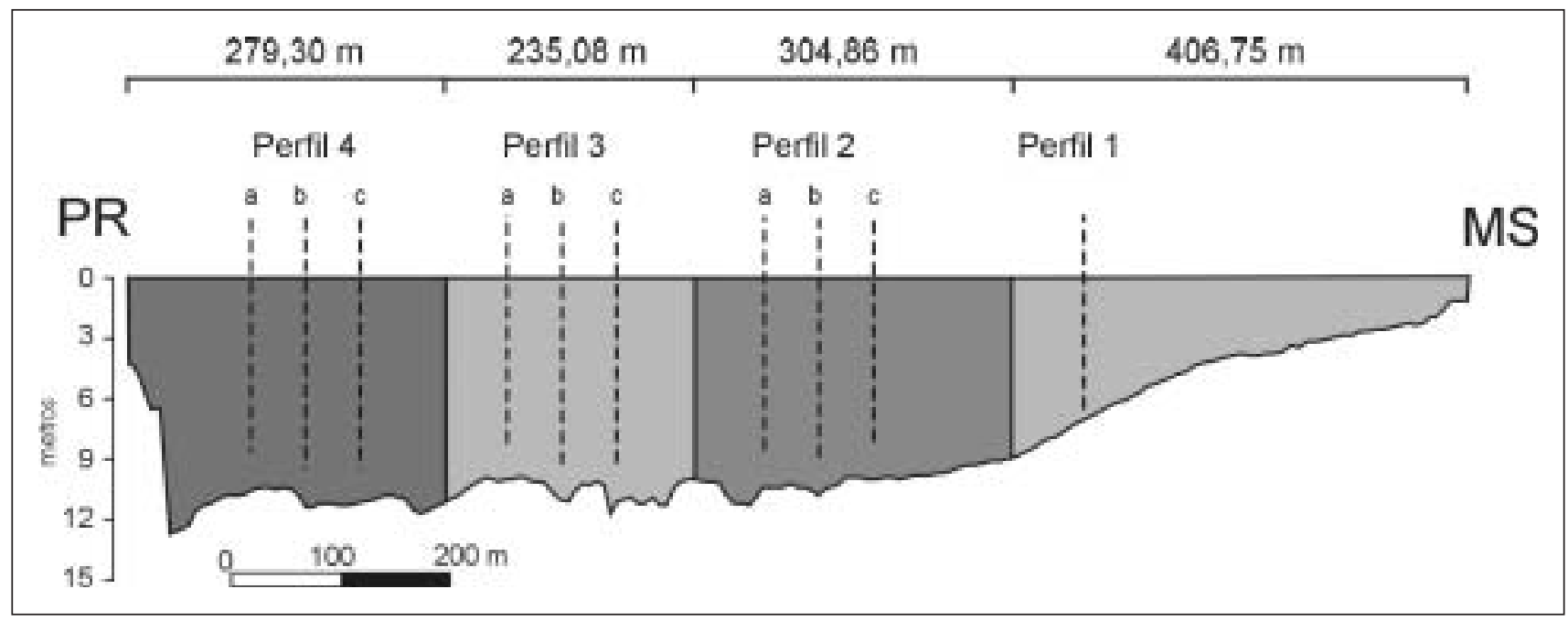

Figura 3: Esquemas dos perfis principais (Perfil 4, 3, 2 e 1) e dos perfis de apoio (a, b e c) para cada perfil proincipal, exceto para o perfil 1. A largura de abrangência dos perfis longitudinais estão representadas por tonalidades diferentes, sendo seus valores dados no topo da figura.

senvolvidas em ocasiões em que a vazão se aproximava da média anual.

O equipamento utilizado para coleta dos dados batimétricos constitui-se de eco-sonda modelo Furuno GP1650F e do Sistema de Posicionamento Global (GPS), acoplados a um computador portátil. A correspondência entre as medições de posição e profundidade forneceu um conjunto de pontos georreferenciados (latitude, longitude, profundidade) a partir dos quais foram determinadas as superfícies batimétricas do fundo do canal.

Na primeira série de levantamentos foram definidos e posicionados com GPS quatro pontos distribuídos ao longo da seção transversal do canal (Figura 3). Cada ponto deu origem a um perfil longitudinal (Perfis 1, 2, 3 e 4 ) com cerca de
$2 \mathrm{~km}$ de extensão obtidos com o barco navegando no sentido da corrente. Para cada perfil batimétrico foram realizados mais dois perfis paralelos equiidistantes cerca de $50 \mathrm{~m}$. Apenas no caso do perfil 1 , devido ausência de formas de leito mais expressivas, não foram realizados os perfis de apoio.

Na segunda série de levantamentos (realizada 16 a 20 dias após a primeira), procurou-se, com auxílio do sistema de navegação por GPS, obter o maior recobrimento possível dos perfis levantados anteriormente. (Figura 5, quadro menor). Na etapa de tratamento de dados, os perfis batimétricos dos dois levantamentos foram lançados em um mesmo gráfico, cada duna foi visualmente identificada, mensurada e codificada numericamente (Figura 5). Durante o tratamento dos dados alguns perfis batimétricos de apoio foram descartados

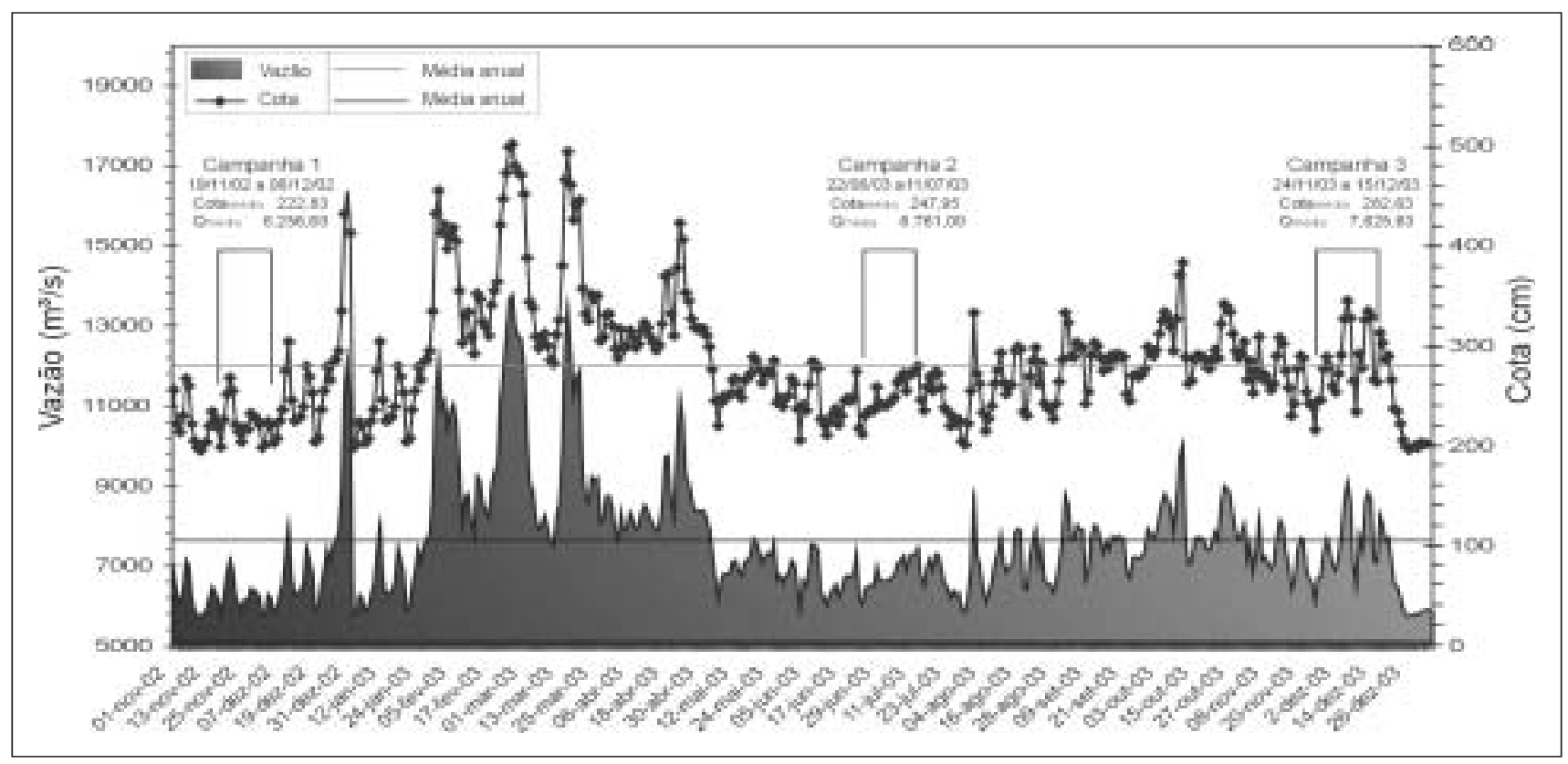

Figura 4: Hidrograma do rio Paraná para o período de estudo. Fonte: ANA. 


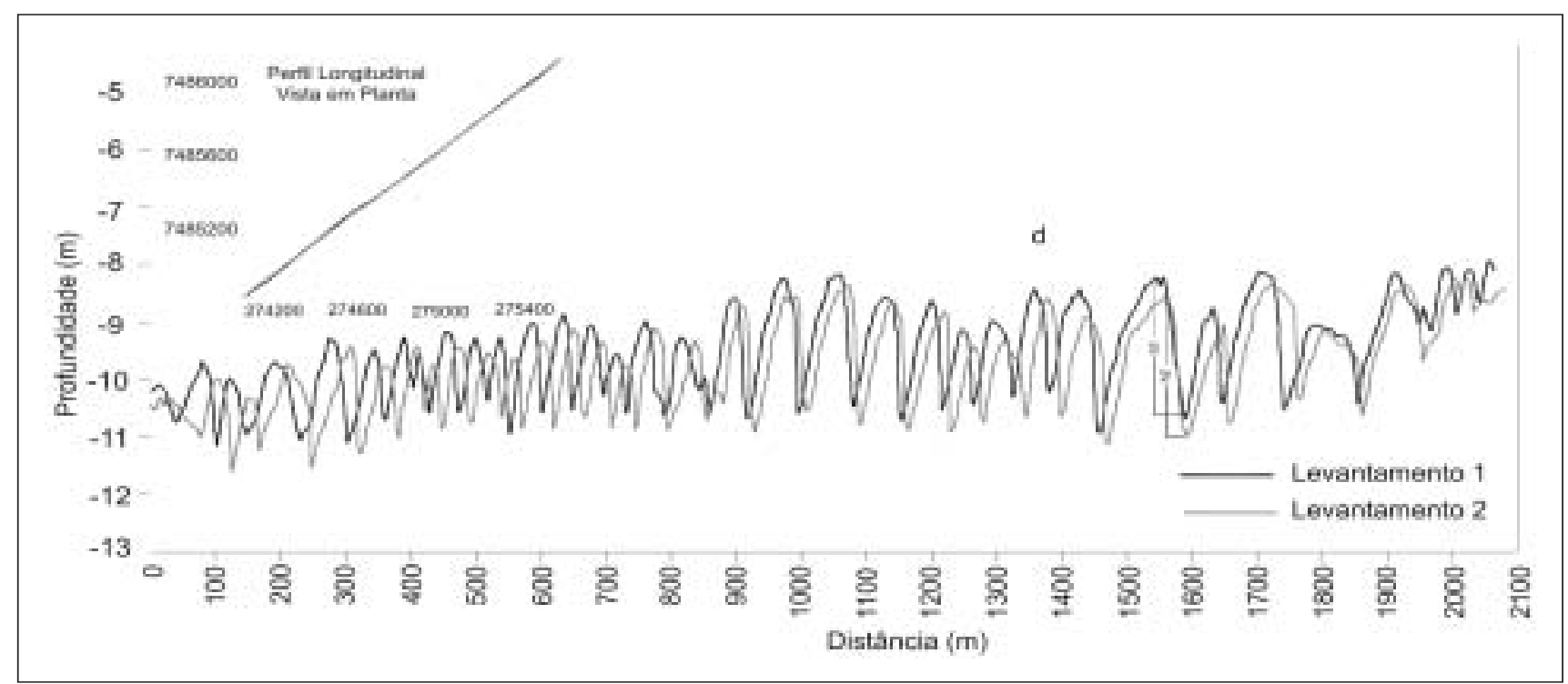

Figura 5: Combinação dos perfis batimétricos obtidos em dois levantamentos. O deslocamento de cada duna é dado por d. No canto esquerdo superior as trajetórias percorridas pelo barco, que no exemplo, apresentam um recobrimento satisfatório.

por não apresentarem uma boa similaridade entre as seqüências de dunas observadas entre os dois levantamentos de uma mesma campanha. A eliminação destes perfis se deu em função de um deslocamento lateral na trajetória do barco, não havendo um recobrimento satisfatório do perfil nos dois levantamentos.

As características geométricas das dunas foram avaliadas a partir da altura e do fator morfométrico das dunas (k). A altura média de cada duna $\left(\mathbf{H}_{\text {pla }{ }^{\prime},{ }^{\prime}, \ldots}, \ldots\right)$ foi medida nos perfis de apoio $\left(\mathbf{H}_{\text {pla,b,c, }},.\right)$ por meio da equação 2, sendo, posteriormente, encontrada a altura média das dunas para cada perfil principal $\left(\mathbf{H}_{\mathrm{pl} 1 \mathrm{p2}, \mathrm{p} 3, \mathrm{p} 4}\right)$ pela equação 4. As dunas encontradas neste estudo apresentaram altura variando entre 0,60 e 2,30m, comprimento (») entre 50 e $150 \mathrm{~m}$. A constante geométrica $(\mathrm{k})$ para dunas com essa morfologia é de 0,66 (STUKRATH, 1969, LIMA et al., 1990 eAMSLER et al., 2000).

A seguir os determinou-se o deslocamento de cada duna $\left(\mathbf{d}_{\mathrm{i}}\right)$, o deslocamento médio das dunas de cada perfil de apoio $\left(\mathbf{d}_{\mathrm{pla}, \mathrm{b}, \mathrm{c}}\right)$ e o deslocamento médio dos referentes aos três perfis de apoio $\left(\mathbf{d}_{\mathrm{pl}}\right)$, utilizando-se respectivamente as equações 3 e 5 . A velocidade média de deslocamento $\left(\mathbf{u d}_{\mathrm{p} 1}\right)$ foi calculada apenas para os perfis principais (Eq. 6).

A partir da equação Stukrath (1969), determinou-se a carga de fundo linear $\left(\mathrm{Cfl}_{\mathrm{P} 1, \mathrm{P} 2, \mathrm{P} 3}\right)$ para cada perfil principal (Eq. 7). O valor encontrado foi posteriormente multiplicado pela sua abrangência lateral $\left(\mathrm{L}_{\mathrm{pl}}\right)$ determinando-se assim a carga de fundo transportada para cada perfil (Eq. 8 e Figura 3). A carga de fundo total $\left(\mathbf{C f}_{\text {total }}\right)$ foi obtida pela soma das $\mathrm{Cf}$ de cada perfil (Equação 9).

O transporte de carga e fundo para a seção do rio Paraná estuda no período, utilizando uma densidade de $2.650 \mathrm{~kg} / \mathrm{m}^{3}$ para a areia, de foi de 2.940 ton/dia na primeira campanha, 2.710 ton/dia, na segunda campanhae 2.812 ton/dia, na terceira campanha(Tabela 1$)$.

Tabela 1: Caracterização das formas de fundo e transporte

\begin{tabular}{|c|c|c|c|c|c|c|c|c|}
\hline & Perfil & $\begin{array}{l}\mathrm{H}_{\mathrm{P}} \\
\mathrm{M}\end{array}$ & $\begin{array}{l}\lambda_{\mathrm{P}} \\
\mathrm{m}\end{array}$ & $\begin{array}{c}\mathrm{ud}_{\mathrm{P}} \\
\mathrm{m} / \mathrm{dia}\end{array}$ & $\begin{array}{c}\mathrm{Cfl}_{\mathrm{P}} \\
\mathrm{m}^{2} / \mathrm{dia}\end{array}$ & $\begin{array}{c}\mathrm{Cf}_{\mathrm{P}} \\
\mathrm{m}^{3} / \mathrm{dia}\end{array}$ & $\begin{array}{c}\mathrm{Cf}^{*}{ }_{\mathrm{P}} \\
\text { ton/dia }\end{array}$ & $\begin{array}{c}\text { Cf*Total } \\
\text { Ton/dia }\end{array}$ \\
\hline \multirow{4}{*}{ 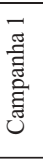 } & P 1 & 0,80 & 98,60 & 1,90 & 0,89 & 385,92 & $1.022,69$ & \multirow{4}{*}{$2.940,40$} \\
\hline & P 2 & 1,31 & 51,75 & 1,89 & 0,91 & 253,13 & 670,78 & \\
\hline & P 3 & 1,20 & 42,24 & 1,70 & 0,90 & 210,79 & 558,59 & \\
\hline & P 4 & 1,66 & 62,21 & 1,85 & 0,94 & 259,75 & 688,33 & \\
\hline \multirow{4}{*}{ 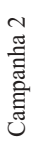 } & P 1 & 0,82 & 96,10 & 1,46 & 0,80 & 351,23 & 930,76 & \multirow{4}{*}{$2.709,61$} \\
\hline & P 2 & 1,57 & 68,44 & 1,60 & 0,81 & 219,75 & 582,33 & \\
\hline & P 3 & 1,45 & 55,56 & 1,48 & 0,99 & 232,73 & 616,73 & \\
\hline & P 4 & 1,79 & 64,41 & 1,30 & 0,78 & 218,79 & 579,78 & \\
\hline \multirow{4}{*}{ 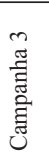 } & P 1 & 0,85 & 99,01 & 1,44 & 0,71 & 307,87 & 815,86 & \multirow{4}{*}{$2.812,38$} \\
\hline & P 2 & 1,40 & 70,39 & 1,06 & 0,85 & 237,36 & 629,01 & \\
\hline & P 3 & 1,20 & 63,09 & 1,56 & 1,05 & 246,05 & 652,03 & \\
\hline & P 4 & 1,50 & 72,34 & 1,70 & 0,97 & 269,99 & 715,47 & \\
\hline
\end{tabular}

Valores para cada perfil principal: $\mathrm{H}_{\mathrm{P}}$ - altura média das dunas, $\mathrm{l}_{\mathrm{P}}$ - comprimento médio das dunas, $\mathrm{ud}_{\mathrm{P}}$ - descolamento linear médio das dunas a jusante, $\mathrm{Cfl}_{\mathrm{P}}$ - carga de fundo linear, $\mathrm{Cf}_{\mathrm{P}}$ - carga de fundo de cada perfil em volume, $\mathrm{Cf}_{\mathrm{P}}^{*}$ - carga e fundo total em cada perfil em peso (para uma densidade da areia de $2.650 \mathrm{~kg} / \mathrm{m}^{3}$. Valores para todo o canal: CF*Total - carga de fundo total da seção em cada campanha. 
Tabela 2: Síntese dos resultados obtidos nas campanhas de campo.

\begin{tabular}{l|c|c|c|c}
\hline & $\begin{array}{c}\boldsymbol{U d} \\
(\mathbf{m} / \text { dia })\end{array}$ & $\begin{array}{c}\boldsymbol{H m} \\
(\boldsymbol{m})\end{array}$ & $\begin{array}{c}\boldsymbol{C f}_{\text {total }} \\
(\text { ton } / \text { dia })\end{array}$ & $\begin{array}{c}\boldsymbol{Q} \\
\left(\boldsymbol{m}^{\mathbf{3} / \text { dia }}\right)\end{array}$ \\
\hline Campanha 1 (nov-dez/2002) & 1,89 & 1,24 & $2.940,40$ & $6.256,5$ \\
\hline Campanha 2 (jun-Ju1/2003) & 1,51 & 1,43 & $2.709,61$ & $6.761,7$ \\
\hline Campanha 3 (nov-dez/2003) & 1,44 & 123 & 281238 & 76250 \\
\hline
\end{tabular}

ud - velocidade média de deslocamento de dunas; $\mathrm{Cf}_{\text {total }}$ - carga de fundo total transportada; $\mathrm{Q}$ - vazão líquida média do período.

Reunindo os valores obtidos nas três campanhas tem-se que durante o período analisado as formas de leito, na seção Porto São José, apresentaram um deslocamento linear médio de 1,6 m/ dia $(47,35 \mathrm{~m} / \mathrm{mês})$ e o transporte médio de carga de fundo foi estimado em 2.820,6 ton./dia, o que corresponde a um total de 1.029.300 ton./ano.

\section{Resultados e Discussão}

Os resultados de velocidade de migração linear da formas de leito e os de descarga sólida obtidos nas três campanhas mostram-se em parte incongruentes quando comparados com a vazão média de cada período (Tabela 2). A descarga de sedimento de fundo observada na campanha 1 foi 4,6\% superior a da campanha 3, para uma vazão líquida $18 \%$ menor. Na campanha 2 a descarga sólida de fundo foi $7,85 \%$ menor que a na primeira para uma descarga líquida $7,45 \%$ maior.

Tal fato, ainda não muito bem compreendido, pode estar relacionado a fatores intrínsecos ao próprio método, como a resolução dos equipamentos tanto na determinação da geometria das dunas (erro do ecobatímetro), como no cálculo da sua velocidade de deslocamento (erro introduzido pelo GPS). Essa afirmativa, embora considerada, é pouco plausível, uma vez que se trata de dados estatísticos provindos de diferentes perfis, em sua maioria compostos por três perfis de apoio, havendo, portanto, diluição do erro.

Outra hipótese é que a discrepância observada esteja relacionada aos períodos de execução das campanhas ao longo do ciclo hidrológico do rio. Durante o período de cheia são mobilizadas as maiores quantidades de sedimento de fundo, com profundas modificações na geometria e na dinâmica local do canal. Stevaux (1994) relatou a destruição de grande parte das formas de leito ("mega-ripples" e dunas) do canal principal do rio Paraná, bem como a remoção de uma grande barra arenosa, após uma cheia excepcional de $1983(\mathrm{Q}=33.740 \mathrm{~m} 3 / \mathrm{s})$. Por outro lado, em algumas porções do canal ocorre intensa sedimentação de carga de fundo, gerando "sand waves" ou mesmo grandes barras arenosas (SANTOS, 1991). Assim, logo após a esses eventos podem localmente ocorrer locais com baixa disponibilidade de sedimento, ou mesmo com transporte sedimentar menos efetivo (menor competência e capacidade). No decorrer do ano, esses tendem, gradativamente, a retornar às suas condições hidrodinâmicas originais. Recentemente Borges (2004) relata alterações na taxa de erosão marginal de ilhas do rio Paraná neste mesmo trecho introduzidas por mudanças na da morfologia do canal (migração lateral do talvegue) após um período de cheia.
No caso estudado, a segunda e a terceira campanha foram realizadas após a cheia de 2003 (Figura4) e apresentaram valores de razãodescarga líquida:descarga sólida de fundo relativamente pró$\operatorname{ximos}(\mathrm{Ql}: \mathrm{Qcf}=2,5 \mathrm{e} 2,7)$. Já a primeira campanha, ocorrida anteriormente à mencionada cheia, apresentou razão Q1:Qcf de 2,1, inferior às outras duas. Dessa forma, considerando o mencionado acima, o trecho estudado durante as campanhas $2 \mathrm{e} 3$, pode ter apresentado características hidráulicas e sedimentares diferentes daquelas da campanha 1 devido alterações provocadas pelas cheias de 2003.

Deve-se também levar em consideração as alterações, ainda desconhecidas e provavelmente não equilibradas introduzidas pelo fechamento da barragem de Porto Primavera. Stevaux \& Takeda (1995)e Crispim (2001), utilizando outra metodologia, apresentaram, para o mesmo trecho do canal, valores de velocidade de deslocamento linear das formas de fundo diferentes dos encontrados neste trabalho. No primeiro caso, Stevaux \& Takeda (op. cit.), analisando um período antecedente ao fechamento da barragem de Porto Primavera (Figura 1), obtiveram uma velocidade de migração de $67 \mathrm{~m} / \mathrm{mês}$, cerca de $17 \%$ superior ao encontrado no presente trabalho. Crispim (2001), por sua vez, que trabalhou no período em que a referida barragem estava sendo fechada, determinou uma velocidade média de deslocamento linear de $62 \mathrm{~m} / \mathrm{mês}$, o que representa, uma redução de 7,5\% em relação à encontrada por Stevaux et al. (1995) e Stevaux \& Takeda (1995) e $9,6 \%$ superior à determinada neste trabalho.

Foram observadas também alterações, não apenas na dinâmica das formas de leito, mas na textura e morfologia do sedimento e na morfologia das formas de leito induzidas pela barragem de Porto Primavera. Considerando os dados de vazão, morfologia das formas de leito e tamanho da partícula transportada, obtidos em trabalhos realizados em períodos anteriores (Stevaux \& Takeda, 1995), concomitantes (Crispim, 2001) e posteriores (Martins \& Stevaux, no prelo) ao fechamento da represa, pode-se supor que as mudanças hidrológicas introduzidas pela barragem de Porto Primavera estejam interferindo tanto na quantidade quanto na dinâmica de transporte de carga de fundo do rio Paraná.

\section{Conclusão}

A quantificação do transporte de fundo num rio com as características do Paraná nãoé uma tarefa fácil de ser realizada. Isso se deve, entre outros fatores, aos erros inerentes às fórmulas e modelagens, que nem sempre são adequadas a rios dessa magnitude. Esta tarefa torna-se ainda mais difícil pelo fato do rio Paraná encontrar-se regulado por uma série de barramentos, e dessa 
forma estar sujeito a modificações na sua geometria hidráulica.

Analisando os resultados obtidos através da metodologia apresentada, os valores obtidos devem ser considerados como uma aproximação, uma vez que parte da carga de fundo se movimenta sob a forma de "ripples" e não é detectada no levantamento batimétrico. Além disso, deve também ser considerado que a fração da carga de fundo que se movimenta por saltação também não é avaliada neste método.

Os resultados obtidos até o momento podem servir como ponto de partida para um monitoramento extensivo deste trecho do rio, possibilitando ainda o desenvolvimento de um modelo teórico no que se refere à mobilidade das formas de leito e ao transporte de sedimentos no setor de estudo, bem como sua aplicação em outros rios da magnitude do rio Paraná. Por se tratar de estudo pioneiro, as estimativas quantitativas aqui apresentadas servem de referência quantitativa e qualitativa, seja pela singularidade do fenômeno estudado, seja pela forma de abordagem.

A grande vantagem da metodologia proposta é a sua aplicação relativamente fácil e a produção de resultados bem mais satisfatórios que outras metodologias empregadas até o momento no rio Paraná. A combinação de dados de carga de fundo com morfologia das formas de leito torna a metodologia apresentada bastante atraente para o estudo do impacto de barragem sobre grandes sistemas fluviais.

\section{Agradecimento}

Ao prof ${ }^{\circ}$ Mário Amsler (UNL - Argentina) pelas críticas e sugestões.

\section{Referências Bibliográficas}

Amsler, M., \& Gaudin, H. E., 1994. La superposición de dunas y el transport de la carga de fondo en el rio Paraná. Memórias XV Congresso Nacional del Água, La Plata, v.3:1-10.

Amsler, M.L., Prendes, H.H., Romano, C.F., et al, 2000. Características de grandes dunas observada en el rio Paraná, XIX Congresso Latinoamericano de Hidráulica, v. I. pp. 329-338, Córdoba, Argentina.

Amsler, M. L., Schreider, M.I., 1999. Dunes height prediction at floods in the Paraná River, Argentina. In: A.W JAYAWARDENA, J.H. LEE \& WANG (eds), River Sedimentation. pp. 615-620. Rotterdam: Balkema.

Borges, C.Z., 2004. Erosão marginal no rio Paraná após a conclusão do reservatório da UHE Engo. Sérgio Motta (Porto Primavera) a jusante da barragem. Dissertação de mestrado UEM/Programa de Pósgraduação em Geografia, Inédito, Maringá, 50p.

Crispim, J. Q., 2001. Alterações na hidrologia do canal após a construção do reservatório a montante: O caso da Usina Hidrelétrica Engenheiro Sérgio Motta (Represa de Porto Primavera) Rio Paraná. Dissertação de mestrado. Universidade
Estadual de Maringá - Pós-Graduação em Ecologia de Ambientes Aquáticos Continentais. 22 p.

Freds фe, J., 1981. Unsteady flow in straight alluvial streams. Part 2. Transition from Dunes to Plane Bed. Journal of Fluid Mechanics, v.102:20 32.

Hubbel, D. W., 1963. Apparatus and techiniques for measuring bedload. U.S. Geol. Survey WaterSuply. Paper 1748.

Itaipu Binacional, 1990. Estudo sobre a carga suspensa na bacia do rio Paraná no período de 1986-1988. Relatório interno s/n. s vol.

Lima, D. R., Campana, N. A., Amsler, M., Schreider, M. I., Gaudin, H. E., 1990. Desplazamiento de dunas y carga de fondo en un tramo del rio Paraná. Memórias XVI congresso Latino-americano de Hidráulica, Montevidéu, Uruguai, v.3:1203-1214.

Orfeo, O., Stevaux, J. C., 2002. Hydraulic and morphological characteristics of middle and upper reaches of the Paraná River (Argentina and Brazil). Geomorphology 44:309-322.

Santos, M.L., 1991. Faciologia e evolução de barras de canal do rio Paraná na região de Porto Rico (PR). Dissertação de mestrado, UNESP, Rio Claro, p.113.

Santos, M.L., Fernandez, O.V.Q. \& Stevaux, J.C., 1992. Aspectos morfogenéticos de barras de canal do rio Paraná, região de Porto Rico. Boletim de Geografia, Maringá, a. 10, n. 1, p. 11-24.

Silva, S.A., 2002. Regime hidrológico e comportamento morfo hidráulico do rio Araguaia. Dissertação de mestrado, UEM, Maringá, 123p.

Simons, D. B., Richardson, E.V., \& Nordin, C. F., 1965. Bedload equation for ripples and dunes. Geol. Survey Prof. Paper 462-H, Washington D.C., 32p.

Souza Filho, E. E., 1993. Aspectos da Geologia e Estratigrafia do rio Paraná entre Porto Primavera (MS) e Guaíra $(P R)$. Tese de Doutoramento. Instituto de Geociências - USP. São Paulo. 214 p.

Stevaux, J. C., 1994. The upper Paraná River (Brazil): Geomorphology, sedimentology and paleoclimatology. Quaternary International, v.21:143-161.

Stevaux, J. C., Takeda, A. M., (1995). Interaction between benthic community and fluvial geomorphological processes inthe upper Paraná River (Brazil). XXVI Congress of International Association of Theoretical and Applied Limnology. São Paulo, Brazil. Abstracts, p 30.

Stevaux, J. C., Takeda, A. M., \& Moraes, M., 1994. Dinâmica sedimentar no canal do rio Paraná. $6^{\circ}$ Simpósio Sul-Brasileiro de Geologia, Porto Alegre, Boletim de Resumos Expandidos, v.1:284-287.

Stuckrath, T.,1969. Movimento de las ondulaciones del lecho de rio Paraná. Mitteilungen das Frazius Institute. Universitat Hannover, v1:20p. (tard. espanhol por Ing. M.H. Balderiote). 